\title{
Radiative decay of heavy-light mesons in a relativistic potential model
}

\section{Takayuki Matsuki}

Tokyo Kasei University, 1-18-1 Kaga, Itabashi, Tokyo 173-8602, JAPAN

E-mail: matsukietokyo-kasei.ac.jp

\section{Koichi Seo*}

Gifu City Women's College, 7-1 Hitoichiba-Kitamachi, Gifu 501-0192, JAPAN

E-mail: seodgifu-cwc.ac.jp

In a relativistic potential model which has succeeded in reproducing the mass spectrum of the heavy-light mesons (charmed or bottom mesons), we have computed the radiative decay widths of the excited heavy-light mesons. The sum of the current quark mass and the constant term in the scalar potential were assumed to be $86 \mathrm{MeV} / c^{2}$ for the up and down quarks and $168 \mathrm{MeV} / c^{2}$ for the strange quark, which are much smaller than the emitted photon energy. In order to take into account the recoil effect of the mesons emitting a photon, we related the wave function of the heavy-light meson in the moving frame to the one in the rest frame. We have evaluated the matrix element of the electro-magnetic current in the Breit frame where the initial and final heavy-light mesons are moving with the velocity $V$ and $-V$ along the $z$-axis, respectively. We included the first order corrections in $1 / m_{Q}$ expansion to the wave function of the mesons. We have obtained the values $11 \mathrm{keV}$ and $0.56 \mathrm{keV}$ for the radiative decay widths of $D^{* 0}$ and $D^{*+}$, respectively. In the non-relativistic arguments, the smallness of the radiative width of $D^{*+}$ compared to the one of $D^{* 0}$ has been explained by the cancellation of the magnetic moments of the constituent quarks. The cancellation is much prominent for $D_{s}^{*+}$ because of the heaviness of the strange quark mass in those arguments. On the other hand, our relativistic formula gives the value $0.72 \mathrm{keV}$ for the radiative decay width of $D_{s}^{*+}$, which is not smaller than that of $D^{*+}$. Large radiative decay widths were obtained for $D_{s J}$ compared with non-relativistic works: $\Gamma\left(D_{s 0}^{*}(2317)^{+} \rightarrow D_{s}^{*+}+\gamma\right)=$ $26 \mathrm{keV}, \Gamma\left(D_{s 1}(2460)^{+} \rightarrow D_{s}^{+}+\gamma\right)=11 \mathrm{keV}, \Gamma\left(D_{s 1}(2460)^{+} \rightarrow D_{s}^{*+}+\gamma\right)=100 \mathrm{keV}$.

Sixth International Conference on Quarks and Nuclear Physics,

April 16-20, 2012

Ecole Polytechnique, Palaiseau, Paris

\footnotetext{
* Speaker.
} 


\section{Lorentz Invariant Evaluation of Transition Amplitudes}

The wave function of a heavy-light meson with a finite momentum is defined as

$$
<0\left|q_{i}^{c}(\vec{x}, t) Q_{j}(\vec{y}, t)\right| P>=\psi_{i j}^{(\xi)}(\vec{x}-\vec{y} ; P) e^{-i P \cdot X_{\xi}},
$$

where $X_{\xi}(=\xi x+(1-\xi) y)$ denotes the position of the heavy-light meson and $\xi$ is a free parameter. If we set $\xi=0$ or 1 , then $X_{\xi}$ coincides with the position of the heavy quark or light quark, respectively.

The electromagnetic interaction of quarks is described by the following Lagrangian,

$$
\mathscr{L}_{i n t}=j_{\mu}^{(e m)} A^{\mu}=\mathscr{L}_{q}+\mathscr{L}_{Q}=\left(-e_{q}\right) \bar{q}^{c} \gamma^{\mu} q^{c} A_{\mu}+e_{Q} \bar{Q} \gamma^{\mu} Q A_{\mu} .
$$

The transition amplitude for one heavy-light meson with a momentum $P$ into another heavylight meson with $P^{\prime}$ by emitting a photon with a momentum $k$ is transformed as follows:

$$
\begin{aligned}
& \mathscr{M}_{P \rightarrow P^{\prime}}= \int d^{4} x<P^{\prime}, k\left|\mathscr{L}_{\text {int }}(x)\right| P> \\
& \approx\left(-e_{q}\right) \int d^{4} x \int d^{3} y \operatorname{tr}\left[\psi^{\prime(\xi) \dagger}\left(\vec{x}-\vec{y} ; P^{\prime}\right) O^{\mu} \psi^{(\xi)}(\vec{x}-\vec{y} ; P)\right] \varepsilon_{\mu} e^{i k \cdot x-i\left(P-P^{\prime}\right) \cdot X_{\xi}} \\
& \quad+e_{Q} \int d^{4} y \int d^{3} x \operatorname{tr}\left[\left(O^{\mu}\right)^{T} \psi^{\prime(\xi) \dagger}\left(\vec{x}-\vec{y} ; P^{\prime}\right) \psi^{(\xi)}(\vec{x}-\vec{y} ; P)\right] \varepsilon_{\mu} e^{i k \cdot y-i\left(P-P^{\prime}\right) \cdot X_{\xi}} \\
&=(2 \pi)^{4} \delta^{4}\left(P-P^{\prime}-k\right) \int d^{3} z\left\{\left(-e_{q}\right) \operatorname{tr}\left[\psi^{\prime(\xi) \dagger}\left(\vec{z} ; P^{\prime}\right) O^{\mu} \psi^{(\xi)}(\vec{z} ; P)\right] e^{-i \vec{k} \cdot \vec{z}(1-\xi)}\right. \\
&\left.\quad+e_{Q} \operatorname{tr}\left[\left(O^{\mu}\right)^{T} \psi^{\prime(\xi) \dagger}\left(\vec{z} ; P^{\prime}\right) \psi^{(\xi)}(\vec{z} ; P)\right] e^{i \vec{k} \cdot \vec{z} \xi}\right\} \varepsilon_{\mu},
\end{aligned}
$$

where $O^{\mu}=\gamma^{0} \gamma^{\mu}$ and $\varepsilon$ stands for the polarization vector of the emitted photon.

In Refs. [四] the following approximate relation has been obtained between the wave function with a finite momentum $\left(P_{3}=\gamma M V\right)$ and the one at rest,

$$
\psi_{\alpha \beta}^{(\xi)}(\vec{x} ; P) \approx G_{\alpha \gamma} G_{\beta \delta} \psi_{\gamma \delta}\left(x_{\perp}, \gamma z ; M\right) e^{i\left\{M(1-\xi)-m_{Q}\right\} V \gamma z},
$$

where $G$ is the boost operator from the rest frame to the moving frame and $\psi(\vec{x} ; M)$ is the wave function in the rest frame. The Lorentz transformation from the rest frame to the moving frame produces the time difference between two constituent quarks, and this time difference is compensated by the free propagation of the heavy quark, which gives rise to the phase factors in Eq.([22).

If we evaluate the transition amplitude in the Breit frame, where the initial heavy-light meson is moving to the $\mathrm{z}$ direction with a velocity $V$ and the final heavy-light meson is moving in the opposite direction with the same velocity $V$, and insert Eq.(L.2) into Eq.(마) we obtain the following expression,

$$
\begin{aligned}
& \mathscr{M}_{P \rightarrow P^{\prime}} \approx(2 \pi)^{4} \delta^{4}\left(P-P^{\prime}-k\right) \\
& \times \gamma^{-1} \int d^{3} x\left\{\left(-e_{q}\right) \operatorname{tr}\left[\psi^{\prime \dagger}\left(\vec{x} ; M^{\prime}\right)\left(G^{-1} O^{\mu} G\right) \psi(\vec{x} ; M)\right] e^{-2 i m_{Q} V z}\right. \\
&\left.+e_{Q} \operatorname{tr}\left[\left(G^{-1} O^{\mu} G\right)^{T} \psi^{\prime \dagger}\left(\vec{x} ; M^{\prime}\right) \psi(\vec{x} ; M)\right] e^{2 i \bar{m}_{q} V z}\right\} \varepsilon_{\mu}
\end{aligned}
$$

where $\bar{m}_{q}=\left(M_{1}+M_{2}\right) / 2-m_{Q}$ and it may be called as the effective mass of the light quark. The free parameter $\xi$ has disappeared in the final expression as it should be. 
In the Breit frame the matrix elements of the electromagnetic current are decomposed into several tensor structures. Some of them are given here:

$$
\begin{aligned}
& \frac{\left\langle 0^{-}\left|j_{\mu}^{(e m)}\right| 1^{-}\right\rangle}{i \sqrt{M_{2} M_{1}}}=\varepsilon_{\mu v \rho \lambda} v_{1}^{v} v_{2}^{\rho} \varepsilon^{\lambda} \xi_{V}^{(1)}, \\
& \frac{\left\langle 1^{-}\left|j_{\mu}^{(e m)}\right| 0^{+}\right\rangle}{i \sqrt{M_{2} M_{1}}}=\left(v_{1} \cdot v_{2}+1\right) \varepsilon_{\mu}^{*} \xi_{V 1}^{(2)}+\left(\varepsilon^{*} \cdot v_{1}\right)\left\{\left(v_{1}+v_{2}\right)_{\mu} \xi_{V 2}^{(2)}+\left(v_{1}-v_{2}\right)_{\mu} \xi_{V 3}^{(2)}\right\},
\end{aligned}
$$

where the initial and final quantities are discriminated by suffices 1 and 2 , respectively. $\varepsilon_{\mu}$ is the polarization vector of the heavy-light meson. $v_{1}$ and $v_{2}$ are velocity vectors defined by $P_{1}=M_{1} v_{1}$ and $P_{2}=M_{2} v_{2}$.

\section{Relativistic potential model and the wave functions}

The Hamiltonian of our potential model in the rest frame of the heavy-light meson is given by

$$
\begin{aligned}
H= & (-i) \vec{\alpha}_{q} \cdot \vec{\nabla}+\beta_{q} m_{q}+i \vec{\alpha}_{Q} \cdot \vec{\nabla}+\beta_{Q} m_{Q} \\
& +\beta_{q} \beta_{Q} S(r)+\left\{1-\frac{1}{2}\left[\vec{\alpha}_{q} \cdot \vec{\alpha}_{Q}+\left(\vec{\alpha}_{q} \cdot \vec{n}\right)\left(\vec{\alpha}_{Q} \cdot \vec{n}\right)\right]\right\} V_{c}(r),
\end{aligned}
$$

where $S(r)=\frac{r}{a^{2}}$ is the confining scalar potential, and $V_{c}(r)=-\frac{4 \alpha_{s}}{3 r}$ is the color Coulomb potential. The constant term in the scalar potential is absorbed into the light quark masses, because the constant term and the light quark masses are not separated from each other at the first order level of $1 / m_{Q}$ expansion.

The wave functions of heavy-light meson in $1 / m_{Q}$ expansion are given by

$$
\psi_{j}(\vec{x} ; M)=U \otimes \frac{\sqrt{2 M}}{\sqrt{4 \pi} r}\left(\begin{array}{c}
u_{j}(r) \\
-i(\vec{n} \cdot \vec{\sigma}) v_{j}(r)
\end{array}\right) y_{j},
$$

where $j$ stands for the quantum numbers to specify the heavy-light meson state. $y_{j}$ 's are the spinangular dependent wave functions and their explicit forms are given in Ref. [[]]. $u_{j}(r)$ and $v_{j}(r)$ are radial wave functions and are solutions of the following Dirac equation,

$$
\left(\begin{array}{cc}
m_{q}+S+V_{c} & -\frac{d}{d r}+\frac{k}{r} \\
\frac{d}{d r}+\frac{k}{r} & -m_{q}-S+V_{c}
\end{array}\right)\left(\begin{array}{c}
u_{j}(r) \\
v_{j}(r)
\end{array}\right)=E_{j}\left(\begin{array}{c}
u_{j}(r) \\
v_{j}(r)
\end{array}\right) .
$$

$U$ denotes the wave function correction and is expanded with respect to $1 / m_{Q}$ (Ref. [B]]). Up to the 1 st order, $U$ is given by

$$
U=1-\frac{1}{2 m_{Q}}\left(\vec{\alpha}_{Q} \cdot \vec{p}\right)+\frac{\alpha_{s}}{3 m_{Q} r}\left[\left(\vec{\alpha}_{Q} \cdot \vec{\alpha}_{q}\right)+\left(\vec{n} \cdot \vec{\alpha}_{Q}\right)\left(\vec{n} \cdot \vec{\alpha}_{q}\right)\right] .
$$

The correction terms are proportional to $\vec{\alpha}_{Q}=\rho_{Q 1} \vec{\sigma}_{Q}$. They survive only if they are multiplied by $\rho_{Q 1}$ in the heavy quark current, because the 0th order wave function does not have the negative energy component with respect to the heavy quark.

The input parameters had been determined to reproduce the mass spectrum of the heavy-light mesons by one of the present author and his collaborators in Refs. [四]. The values of them are shown in Table 1. 
Table 1: Values of the parameters to be used

\begin{tabular}{|c|c|c|c|c|c|}
\hline$\alpha_{s}$ & $\begin{array}{c}a \\
\left(\mathrm{GeV}^{-1}\right)\end{array}$ & $\begin{array}{c}b+m_{u}\left(=m_{d}\right) \\
\left(\mathrm{MeV} / c^{2}\right)\end{array}$ & $\begin{array}{c}b+m_{s} \\
\left(\mathrm{MeV} / c^{2}\right)\end{array}$ & $\begin{array}{c}m_{c} \\
\left(\mathrm{MeV} / c^{2}\right)\end{array}$ & $\begin{array}{c}m_{b} \\
\left(\mathrm{MeV} / c^{2}\right)\end{array}$ \\
\hline $\begin{array}{c}0.259(D) \\
0.392(B)\end{array}$ & 1.937 & 86 & 168 & 1023 & 4634 \\
\hline
\end{tabular}

\section{Transition amplitudes in terms of the radial wave functions and numerical results}

Now the transition amplitudes are expressed in terms of the radial wave functions. The formulas for (1) $1^{-} \rightarrow 0^{-}$and (2) $0^{+} \rightarrow 1^{-}$are shown here as an example of the M1 transition and the E1 transition, respectively, and formulas for other processes will be published in the preparing article. (1) $1^{-}\left({ }^{3} S_{1}\right) \rightarrow 0^{-}\left({ }^{1} S_{0}\right)$

$$
\begin{aligned}
\eta^{(1)} \equiv V \xi_{V}^{(1)} / e= & \int d r\left\{-f_{q}^{\prime}\left(u_{2} v_{1}+v_{2} u_{1}\right)+V\left(f_{q} u_{2} u_{1}+f_{q}^{\prime \prime} v_{2} v_{1}\right)+V f_{Q}\left(u_{2} u_{1}+v_{2} v_{1}\right)\right\} \\
& +\frac{1}{m_{Q}} \int d r\left\{\frac{p}{2}\left(f_{Q} u_{2} u_{1}-f_{Q}^{\prime \prime} v_{2} v_{1}\right)+\frac{2 \alpha_{s} f_{Q}^{\prime}}{3 r}\left(u_{2} v_{1}+v_{2} u_{1}\right)\right\} .
\end{aligned}
$$

where $V$ is the velocity of the heavy-light meson in the Breit frame. $f_{q}$ and $f_{Q}$ are defined by $f_{q}=\frac{e_{q}}{e} f(q r)$ and $f_{Q}=\frac{e_{Q}}{e} f(p r)$, where $q=-2 m_{Q} V$ and $p=2 \bar{m}_{q} V . f(x)$ is the 0th order spherical Bessel function, that is, $f(x) \equiv \sin x / x$. By averaging over the initial polarization vectors, we obtain the radiative decay width as

$$
\Gamma=\frac{4 \alpha M_{2}}{3 M_{1}} k\left(\eta^{(1)}\right)^{2},
$$

where $k$ is the photon momentum and $\alpha$ is the fine structure constant.

(2) $0^{+}\left({ }^{3} P_{0}\right) \rightarrow 1^{-}\left({ }^{3} S_{1}\right)$

$$
\begin{aligned}
& \eta^{(2)} \equiv \xi_{V 1}^{(2)} / e=\int d r\left\{f_{q} u_{2} v_{1}-f_{q}^{\prime \prime} v_{2} u_{1}+V f_{q}^{\prime}\left(u_{2} u_{1}-v_{2} v_{1}\right)-V f_{Q}^{\prime}\left(u_{2} u_{1}+v_{2} v_{1}\right)\right\} \\
& +\frac{1}{2 m_{Q}} \int d r\left\{\left(f_{Q}+f_{Q}^{\prime \prime}\right)\left(u_{2} u_{1}^{\prime}+v_{2} v_{1}^{\prime}\right)-\frac{2 f_{Q}^{\prime \prime}+p r f_{Q}^{\prime}}{r} u_{2} u_{1}-\frac{f_{Q}+f_{Q}^{\prime \prime}+p r f_{Q}^{\prime}}{r} v_{2} v_{1}\right. \\
& \left.-\frac{2 \alpha_{s}\left(3 f_{Q}+f_{Q}^{\prime \prime}\right)}{3 r} u_{2} v_{1}+\frac{2 \alpha_{s}\left(f_{Q}+3 f_{Q}^{\prime \prime}\right)}{3 r} v_{2} u_{1}\right\}
\end{aligned}
$$

By summing up the final polarization vectors, we obtain the radiative width as

$$
\Gamma=\frac{4 \alpha M_{2}}{M_{1}} k\left(\eta^{(2)}\right)^{2}
$$

Our numerical results for the radiative decay widths are shown in Tables 2 and 3. $\pi^{0}$ emitting decay widths are cited from our previous work (Refs. []ㅣ). We also present the 0th order radiative decay widths without wave function corrections in the square bracket. The experimental values are cited from Ref. [[]].

The radiative decay width of $D^{*+}$ becomes very small compared with that of $D^{* 0}$ if we do not include the 1 st order wave function corrections. The width of $D_{s}^{*+}$ is also largely affected by 
Table 2: Radiative decay widths of the excited $D$ and $B$ mesons

\begin{tabular}{|l|c|c|c|c|c|}
\hline $\begin{array}{c}\text { Initial State } \\
\left({ }^{2 S+1} L_{J}\right)\end{array}$ & Final State & $\begin{array}{c}\Gamma_{t h}\left(\pi^{0}\right) \\
(\mathrm{keV})\end{array}$ & $\begin{array}{c}\Gamma_{t h}(\gamma) \\
(\mathrm{keV})\end{array}$ & $\left(\gamma / \pi^{0}\right)_{t h}$ & $\left(\gamma / \pi^{0}\right)_{\exp }$ \\
\hline \hline$D^{* 0}\left({ }^{3} S_{1}\right)$ & $D^{0}$ & 42 & $11[6.4]$ & 0.26 & 0.62 \\
\hline$D^{*+}$ & $D^{+}$ & 29 & $0.56\left[4.9 \times 10^{-4}\right]$ & 0.019 & 0.052 \\
\hline$D_{0}^{*}(2400)^{0}\left({ }^{3} P_{0}\right)$ & $D^{* 0}$ & $5.0 \times 10^{4}$ & $282[49]$ & $5.6 \times 10^{-3}$ & \\
\hline$D_{0}^{*}(2400)^{+}$ & $D^{*+}$ & $6.1 \times 10^{4}$ & $51[8.1]$ & $8.4 \times 10^{-4}$ & \\
\hline$D_{1}(2430)^{0}\left({ }^{\prime 3} P_{1} "\right)$ & $D^{0}$ & 0 & $148[21]$ & & \\
& $D^{* 0}$ & $3.9 \times 10^{4}$ & $121[37]$ & $3.1 \times 10^{-3}$ & \\
\hline$D_{1}(2430)^{+}$ & $D^{+}$ & & $16[13]$ & & \\
& $D^{*+}$ & & $104[10]$ & & \\
\hline$D_{1}(2420)^{0}\left(" 1 P_{1} "\right)$ & $D^{0}$ & 0 & $559[180]$ & & \\
& $D^{* 0}$ & $1.5 \times 10^{3}$ & $159[95]$ & 2.11 & \\
\hline$D_{1}(2420)^{+}$ & $D^{+}$ & 0 & $20[31]$ & & \\
\hline \hline$B^{* 0}\left({ }^{3} S_{1}\right)$ & $D^{*+}$ & $1.4 \times 10^{3}$ & $41[13]$ & $2.9 \times 10^{-2}$ & \\
\hline$B^{*+}$ & $B^{0}$ & & $4.6 \times 10^{-2}\left[4.4 \times 10^{-2}\right]$ & & \\
\hline$B_{0}^{*}(5590)^{0}\left({ }^{3} P_{0}\right)$ & $B^{* 0}$ & $1.7 \times 10^{4}$ & $24[15]$ & $1.4 \times 10^{-3}$ & \\
\hline$B_{0}^{*}(5590)^{-}$ & $B^{*-}$ & & $47[61]$ & & \\
\hline$B_{1}(5646)^{0}\left(" 3 P_{1} "\right)$ & $B^{0}$ & 0 & $12[8.2]$ & & \\
& $B^{* 0}$ & $1.9 \times 10^{4}$ & $14[13]$ & $7.4 \times 10^{-4}$ & \\
\hline$B_{1}(5646)^{-}$ & $B^{-}$ & & $26[33]$ & & \\
\hline$B_{1}(5721)^{0}\left(" 1 P_{1} "\right)$ & $B^{*-}$ & & $55[54]$ & & \\
\hline$B_{1}(5721)^{-}$ & $B^{* 0}$ & $3.8 \times 10^{3}$ & $22[19]$ & $5.8 \times 10^{-3}$ & \\
\hline & $B^{*-}$ & 0 & $108[140]$ & & \\
\hline
\end{tabular}

the wave function corrections. In the case of $B_{s}^{* 0}$ the contribution of the wave function corrections is negligible. In the non-relativistic framework (Refs. [目]), the smallness of the radiative decay width of $D^{*+}$ compared with that of $D^{* 0}$ is explained as follows. Each quark is assumed to have a magnetic moment $\frac{e_{j}}{m_{j}^{*}} \vec{s}_{j}$, where $m_{j}^{*}$ denotes the constituent quark mass. For the process $1^{-} \rightarrow 0^{-}$, the total magnetic moment becomes $\frac{e_{q}}{m_{q}^{*}}+\frac{e_{Q}}{m_{Q}^{*}}$. In the case of $D^{*+}$, two terms cancel with each other and the net magnetic moment becomes small. On the other hand, our relativistic formulas do not allow such an interpretation, and this point will be briefly discussed in Sec. 4 .

\section{Discussion}

Comparing our relativistic results with non-relativistic results by other authors in Refs. [四], the former predicts fairly larger values for the decay widths of $D_{s J}$ than the latter. For example, we give the values $26 \mathrm{keV}$ and $100 \mathrm{keV}$ for $\Gamma\left(D_{s 0}^{*}(2317)^{+} \rightarrow D_{s}^{*+}+\gamma\right)$ and $\Gamma\left(D_{s 1}(2460)^{+} \rightarrow D_{s}^{*+}+\gamma\right)$, respectively. Meanwhile non-relativstic calculations give at most $1.9 \mathrm{keV}$ and $5.6 \mathrm{keV}$, 
Table 3: Radiative decay widths of the excited $D_{s}$ and $B_{s}$ mesons

\begin{tabular}{|c|c|c|c|c|c|}
\hline $\begin{array}{c}\text { Initial State } \\
\left({ }^{2 S+1} L_{J}\right) \\
\end{array}$ & Final State & $\begin{array}{c}\Gamma_{t h}\left(\pi^{0}\right) \\
(\mathrm{keV}) \\
\end{array}$ & $\begin{array}{r}\Gamma_{t h}(\gamma) \\
(k e V)\end{array}$ & $\left(\gamma / \pi^{0}\right)_{t h}$ & $\left(\gamma / \pi^{0}\right)_{\exp }$ \\
\hline$D_{s}^{*+}\left({ }^{3} S_{1}\right)$ & $\overline{D_{s}^{+}}$ & $8.0 \times 10^{-3}$ & $0.72\left[2.8 \times 10^{-3}\right]$ & 90 & 16 \\
\hline $\begin{array}{l}D_{s 0}^{*}(2317)^{+} \\
\left({ }^{3} P_{0}\right)\end{array}$ & $\begin{array}{c}D_{s}^{+} \\
D_{s}^{*+}\end{array}$ & $\begin{array}{c}3.8 \\
0\end{array}$ & $\begin{array}{c}0 \\
26[12]\end{array}$ & 6.8 & $<0.059$ \\
\hline $\begin{array}{l}D_{s 1}(2460)^{+} \\
\left(" 3 P_{1} "\right)\end{array}$ & $\begin{array}{c}D_{s}^{+} \\
D_{s}^{*+}\end{array}$ & 3.9 & $\begin{array}{c}11[16] \\
101[16]\end{array}$ & 26 & \\
\hline $\begin{array}{l}D_{s 1}(2536)^{+} \\
\left({ }^{\prime 3} P_{1} "\right)\end{array}$ & $\begin{array}{c}D_{s}^{+} \\
D_{s}^{*+}\end{array}$ & & $\begin{array}{l}27[32] \\
48[13]\end{array}$ & & \\
\hline$B_{s}^{*}\left({ }^{3} S_{1}\right)$ & $\overline{B_{s}}$ & not allowed & $4.2 \times 10^{-2}\left[4.0 \times 10^{-2}\right]$ & & \\
\hline $\begin{array}{l}B_{s 0}^{*}(5615)^{0} \\
\left({ }^{3} P_{0}\right)\end{array}$ & $\begin{array}{l}B_{s} \\
B_{s}^{*}\end{array}$ & $\begin{array}{c}1.6 \\
0\end{array}$ & $\begin{array}{c}0 \\
30[21]\end{array}$ & 19 & not found \\
\hline $\begin{array}{l}B_{s 1}(5679)^{0} \\
\left(" 3 P_{1} "\right)\end{array}$ & $\begin{array}{l}B_{s} \\
B_{s}^{*}\end{array}$ & $\begin{array}{c}0 \\
1.9\end{array}$ & $\begin{array}{l}16[12] \\
20[19]\end{array}$ & 11 & not found \\
\hline $\begin{array}{l}B_{s 1}(5830)^{0} \\
\left({ }^{3} P_{1} "\right)\end{array}$ & $\begin{array}{l}B_{s} \\
B_{s}^{*}\end{array}$ & 0 & $\begin{array}{l}53[35] \\
23[19]\end{array}$ & & not found \\
\hline
\end{tabular}

respectively(Refs. [甘]). According to the non-relativistic arguments, the amplitude of E1 transition is proportional to the mass weighted electric dipole moment $\frac{e_{q} m_{Q}^{*}+e_{Q} m_{q}^{*}}{m_{Q}^{*}+m_{q}^{*}}<r>$. As the M1 transition discussed in the previous section, two terms cancel with each other in the case of $D_{S J}$. On the other hand, our relativistic expression for the light quark contribution (1st and 2nd terms in Eq. (B.3)) can be reduced to the electric dipole moment by making use of the Dirac equation ([2.3), but the heavy quark contribution (the terms proportional to $1 / m_{Q}$ in Eq. (3.3])) cannot be transformed into an electric dipole moment due to the existence of the confining scalar potential. These points will be discussed in more detail in a future publication.[[]]

\section{References}

[1] T. Matsuki and K. Seo, Prog. Theor. Phys. 118, 1087 (2007); Bulletin of Gifu City Women's College No.47, 9 (1998).

[2] T. Matsuki and K. Seo, Phys. Rev. D 85, 014036 (2012); T. Matsuki, "Chiral particle decay of heavy-light mesons in a relativistic potential model", a talk given in the poster session of this conference (QNP2012).

[3] T. Matsuki and K. Seo, Bulletin of Gifu City Women's College No.46, 7 (1997).

[4] T. Matsuki and T. Morii, Phys. Rev. D56, 5646 (1997); T. Matsuki, T. Morii, and K. Sudoh, Prog. Theor. Phys. 117, 1077 (2007); Eur. Phys. J. A31, 701 (2007).

[5] K. Nakamura et al. (Particle Data Group), Journal of Physics G 37, 075021 (2010).

[6] W. A. Bardeen, E. J. Eichten and C. T. Hill, Phys. Rev. D 68, 054024 (2003); F. E. Close and E. S. Swanson, Rev. D 72, 094004 (2005); S. Godfrey, Rev. D 72, 054029 (2005).

[7] T. Matsuki and K. Seo, paper in preparation. 